Research Article

\title{
Dynamic Modeling and Analysis of Multidimensional Hybrid Recommendation Algorithm in Tourism Itinerary Planning under the Background of Big Data
}

\author{
Yange $\mathrm{Hao}^{1}$ and $\mathrm{Na}$ Song $\mathbb{D}^{2}$ \\ ${ }^{1}$ Hebei Construction Material Vocational and Technical College, Qinhuangdao, Hebei, China \\ ${ }^{2}$ School of Economics and Management, Yanshan University, Qinhuangdao 066004, China \\ Correspondence should be addressed to Na Song; songna129412@ysu.edu.cn
}

Received 23 October 2021; Revised 10 November 2021; Accepted 23 November 2021; Published 15 December 2021

Academic Editor: Gengxin Sun

Copyright (c) 2021 Yange Hao and Na Song. This is an open access article distributed under the Creative Commons Attribution License, which permits unrestricted use, distribution, and reproduction in any medium, provided the original work is properly cited.

\begin{abstract}
Smart tourism can provide high-quality and convenient services for different tourists, and tourism itinerary planning system can simplify tourists' tourism preparation. In order to improve the limitation of the recommendation dimension of traditional travel planning system, this paper designs a mixed user interest model on the premise of traditional user interest modeling and combines various attributes of scenic spots to form personalized recommendation of scenic spots. Then, it uses heuristic travel planning cost-effective method to construct the corresponding travel planning system for travel planning. In terms of the accuracy rate of travel planning recommendation, the accuracy rate of multidimensional hybrid travel recommendation algorithm is 0.984 , and the missing rate is 0 . When the travel cost and travel time are the same and the number of scenic spots is $20-30$, the memory occupation of $\mathrm{MH}$ algorithm is only $1 / 2$ of that of TM algorithm. The results show that the multidimensional hybrid travel recommendation algorithm can improve the personalized travel planning of users and the travel time efficiency ratio. The results of this study have a certain reference value in improving user satisfaction with the travel planning system and reducing user interaction.
\end{abstract}

\section{Introduction}

Smart tourism is to actively perceive the information of tourism resources, tourism economy, tourism activities, and tourists by using new technologies such as cloud computing and the Internet of things, through the Internet/mobile Internet and with the help of portable terminal Internet access equipment. Through timely release, people can understand this information and arrange and adjust work and tourism plans in a timely manner, so as to achieve the effect of intelligent perception and convenient utilization of all kinds of tourism information. The construction and development of smart tourism will eventually be reflected in three levels: tourism management, tourism service, and tourism marketing. It is based on the integrated communication and information technology, centered on the interactive experience of tourists, guaranteed by the integrated industry information management, and characterized by encouraging industrial innovation and promoting the upgrading of industrial structure. Smart tourism is to actively perceive tourism related information by using new technologies, such as mobile cloud computing and the Internet, and portable terminal Internet devices.

The development of the Internet promotes the improvement of the service quality of the tourism industry, and smart tourism provides better services for tourists [1]. With the development of intelligent mobile device positioning technology, users can plan their travel itinerary according to the tourism related recommendation system [2]. However, the traditional recommendation technology cannot meet the needs of the tourism recommendation system, because the user model of the traditional recommendation technology is very simple, in addition to the lack of full use of user information. Furthermore, the recommendation dimension is 
single; unable to meet the needs of tourism recommendation in terms of location, user status, tourism restrictions, etc.; and unable to achieve the user's personalized recommendation needs. Moreover, in the organization and purpose of tourism destination, there are some deficiencies in the way of land planning $[3,4]$. Therefore, to explore a more effective and comprehensive travel recommendation algorithm is of great significance for improving users' satisfaction with the travel recommendation system, reducing user interaction, enhancing the scientific and effective travel planning, and meeting the personalized needs of users.

On the premise of traditional user interest modeling, this paper designs a hybrid user interest model and combines various attributes of scenic spots to form personalized recommendation of scenic spots. The innovations of this paper include the following: 1 . It can provide high-quality and convenient services for different tourists, and the tourism itinerary planning system can simplify tourists' tourism preparation. 2. Multidimensional hybrid travel recommendation algorithm can improve users' personalized travel planning and travel time efficiency. 3. It has certain reference value for improving users' satisfaction with the travel planning system and reducing user interaction.

This paper is divided into five sections. Section $1 \mathrm{ex}-$ pounds the current research background of intelligent tourism. The development of Internet promotes the improvement of tourism service quality. Section 2 expounds the content of relevant literature research. Section 3 describes the design of multidimensional hybrid travel route recommendation algorithm. A hybrid user interest model is established, and a heuristic travel planning algorithm is designed. Section 4 verifies the performance of multidimensional hybrid travel recommendation algorithm and analyzes the application effect of tourism route planning system. Finally, the full text is summarized in Section 5.

\section{Related Work}

With the improvement of science and technology, more and more people pay attention to air travel planning. Liu et al. propose an air travel planning algorithm based on user preference characteristics. Users can obtain efficient air travel planning scheme by calling the API provided by airlines [5]. An excellent tourism itinerary planning scheme can greatly improve the time utilization rate of tourists. Yochum et al. proposed an adaptive genetic algorithm based on cross mutation probability, which gives different weights to different tourists' travel needs and realizes personalized travel recommendation of tourists [6]. In recent years, more and more research has proposed a variety of travel package recommendation algorithms to improve the service of online travel agency. Zhu et al. established a new tourism package recommendation model by combining the longterm preference and short-term preference of users through the gating fusion method and carried out experimental verification. The experimental results show that this model can push more accurate tourism itinerary planning scheme to users [7]. Jiao et al. proposed an adaptive learning rate function combining exponential function with linear function to optimize the recommendation algorithm based on singular value decomposition (SVD)++, shorten its modeling time, and improve the operation efficiency of the algorithm [8].

The modern development of Internet technology has attracted researchers to explore personalized location-based service recommendation system. Logesh et al. have designed a travel recommendation system based on mixed location. The system uses integrated collaborative training method and swarm intelligence algorithm to improve personalized travel recommendation. The experimental results show that the system can effectively improve user satisfaction [9]. The existing tourism websites rarely support the collaborative information search activities of tourists, so Arif et al., on the basis of investigating people's previous research results on collaborative tourism information search behavior, proposed a collaborative search system supporting online information search and tourism planning. The system has the functions of collaborative query, reformulation, and automatic sharing of query results and realizes the exchange and comparison of vacation information among multiple people [10]. Qu et al. established a probabilistic network model by using Kalman filtering method to predict the pick-up probability and capacity of each position on the way to travel, and recommend the optimal driving route to the driver. At the same time, the shortest expected cruise distance was introduced into the model to improve the recommendation efficiency of the scheme [11]. Lyu et al. believe that the existing travel recommendation system cannot deal with different information specifically, so they propose a weighted multi-information constraint matrix decomposition scheme for personalized travel location recommendation based on geographical tag photos, to achieve a comprehensive description of users' travel needs from the perspective of photos, user access sequences, text tags, and so on [12].

To sum up, the relevant researchers have made good achievements in tourism itinerary planning, driving route planning, personalized travel itinerary setting technology, etc., but the designed travel itinerary recommendation algorithm is mostly a single algorithm, which does not make full use of the information that tourists have, and there are still some shortcomings in personalized travel itinerary planning technology. Therefore, a tourism itinerary planning technology based on multidimensional hybrid recommendation algorithm is studied and designed, including hybrid user interest modeling and targeted tourism itinerary planning algorithm by introducing multidimensional scenic spot scoring. Taking this technology as the core, a tourism itinerary planning system is designed.

\section{Design of Multidimensional Hybrid Travel Itinerary Recommendation Algorithm}

3.1. Hybrid User Interest Model. Tourism user's interest model has the characteristics of structure, stability, and real time. Stability means that specific noise data will not interfere with the results of the interest model; real time means that the user's interest information can be dynamically 
updated to express the user's interest preferences in real time $[13,14]$.

The acquisition of user interest, characteristics, and other related data is the premise of establishing user interest model, and the accurate description of user browsing behavior and interest data is the basis of constructing efficient user interest model [15]. As shown in Figure 1, user information is divided into static information and dynamic information, which can be obtained explicitly or implicitly. Static information is composed of user's gender, name, occupation, education background, contact information, and other parts, which cannot accurately provide user's interest needs; dynamic information is mainly obtained by implicit way, and relevant information is extracted by user's historical browsing behavior and dynamic information, which has certain dynamic and redundancy [16].

As shown in Figure 2, the user interest model is divided into short-term interest and long-term interest. The shortterm user interest model contains the user's recent interest items according to the user's implicit information (click events), which has immediate attributes; the long-term user interest model is based on the user's static information and short-term interest, which contains the user's always interested items, which has cumulative attributes $[14,17]$. The user interest model is used to express the user's interest in a certain item, and the user interest is defined by the expression based on space vector.

$$
U_{i}=\left\{\left(I_{1}, W_{1}, T_{1}\right),\left(I_{2}, W_{2}, T_{2}\right), \ldots,\left(I_{n}, W_{n}, T_{n}\right)\right\},
$$

$U_{i}$ is the definition of user demand model, where triple $(I, W, T)$ represents the feature item of user interest model; $I$ refers to the item of user interest; user's interest value of item $I$ is $W$; the last update time of item interest value is $T$. According to the user's scenic spot $j$ saving behavior $S(j)$, the number of scenic spot visits $F(j)$, and the corresponding scenic spot page view time $D(j)$, the interaction behavior between the user and the scenic spot $j$ is analyzed.

$$
S(j)=\left\{\begin{array}{l}
1, \\
0 .
\end{array}\right.
$$

Equation (2) shows that when the user saves, $S(j)=1$; when the user does not save, $S(j)=0$.

$$
F(j)= \begin{cases}0, & f>f_{0}, \\ \partial f, & f \leq f_{0} .\end{cases}
$$

In equation (3), $f$ is the orientation times of the user to the scenic spot page, and the preset access threshold is $f_{0}$; when the number of visits is greater than $f_{0}$, it is regarded as an access exception; on the contrary, the number of visits is weighted to obtain $F(j)$. The longer the page viewing time is, the higher the user's interest in the scenic spot is. Considering the number of words on the page of the scenic spot, the viewing time $t$ of the average number of words on the page is calculated by the number of words on the page.

When the page viewing time is too long, the user may not view the page as noise data.

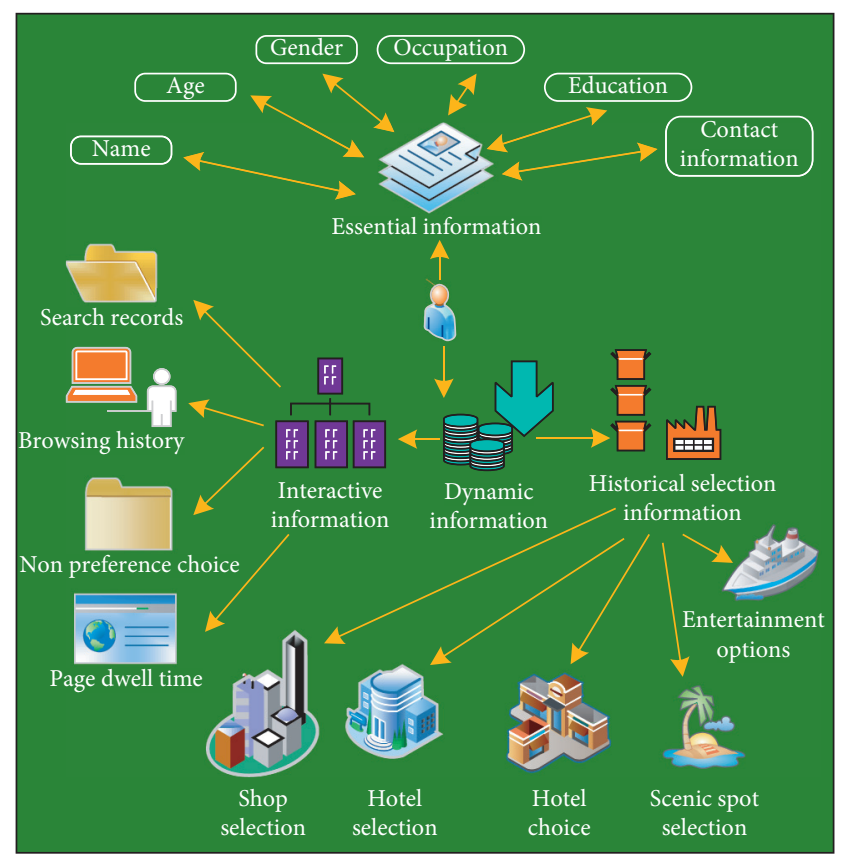

FIgURE 1: Tourist user information collection.

$$
D(j)= \begin{cases}0, & t>t_{0}, \\ \frac{\varepsilon t}{M}, & t \leq t_{0} .\end{cases}
$$

In equation (4), $\varepsilon$ and $M$ are the weighting coefficient and the total number of words on the scenic spot page. Combining the above three behaviors, we can get the shortterm interest formula of users.

$$
U^{\text {cur }}(j)=\alpha S(j)+\beta F(j)+\gamma D(j)+c .
$$

In equation (5), $\alpha, \beta, \gamma, c$ are constants, which are mainly used to adjust the influence coefficient of each of the three user interaction behaviors on the short-term interest model according to the system requirements. The short-term user interest model reflects the rapid change of user interest. The drift processing of this model should have the advantage of fast response, which can be realized by maintaining the short-term interest sliding window with the size of $n^{\text {cur }}$.

$$
U^{\text {cur }}=\left\{\left(I_{1}^{\text {cur }}, W_{1}^{\text {cur }}, F_{1}\right),\left(I_{2}^{\text {cur }}, W_{2}^{\text {cur }}, F_{2}\right), \ldots,\left(I_{n}^{\text {cur }}, W_{n}^{\text {cur }}, F_{n}\right)\right\} \text {. }
$$

In equation (6), judge whether the short-term interest scenic spot is in the sliding window by the frequency $F$ of the item. When it is in the sliding window, add 1 to the frequency $F$ of the corresponding item; if it is not in the sliding window, remove the item with the lowest frequency in the sliding window $U^{\text {cur }}$. Long-term user interest reflects the user's persistent interest item, and the interest value of this item will be forgotten or changed by the user over time. Therefore, the drift strategy of long-term user interest is formulated from the two aspects of forgetting strategy and incremental strategy [18]. 


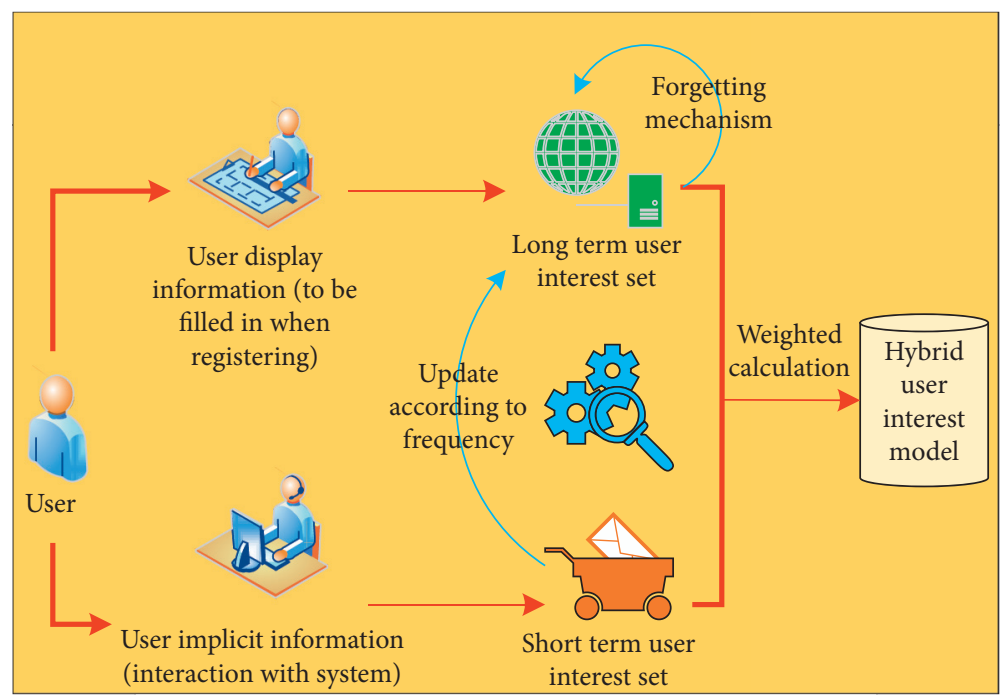

Figure 2: The process of building hybrid user interest model.

$$
W_{i}=\frac{1}{\alpha \sigma \sqrt{2 \pi}}^{-i^{2} / e^{2}(\beta \sigma)^{2}} .
$$

Equation (7) is a nonlinear forgetting function. Let $\alpha$ be 0.52 and $\beta$ be 2 ; let $i$ denote each item in the long-term interest model $U^{\text {cur }}, i \in[1, n]$, and $n$ denote the number of interesting items in the long-term interest model, arranged according to the update time of each item, so that the latest updated item corresponding to $i$ is 1 .

As shown in Figure 3, incremental strategy is a strategy to update long-term user interests with accumulated shortterm interests. In the process of short-term interest updating, a frequency count $F_{j}$ corresponds to $\left(I_{j}^{\text {cur }}, W_{j}^{\text {cur }}, F_{j}\right)$ in the sliding window. When $F_{j}$ is greater than the preset threshold $F_{0}$, the corresponding short-term interest is accumulated into a long-term model to achieve the purpose of updating the long-term model. If there is an item $I_{j}$ in the long-term interest model, the short-term interest model is used to update the interest value and update the corresponding timestamp; when there is no item $I_{j}$ in the long-term interest model, the last item in the long-term interest queue is removed, and $I_{j}$ is added to the long-term interest model [19].

$$
U=\alpha U^{\text {pur }}+\beta U^{\text {pur }}
$$

Formula (8) combines the short-term interest model with the long-term interest model and calculates the user interest model by weighting interests. Among them, $\alpha+\beta=1,0<\alpha, \beta<1$, the weight of short-term interest in user interest model is $\alpha$, and the weight of long-term interest in user interest model is $\beta$.

3.2. Heuristic Travel Planning Algorithm Design. The heuristic travel planning algorithm is used to plan the travel route. Considering the network form and line departure frequency, a public transport network design method (nonsingle route design method)-heuristic public transport

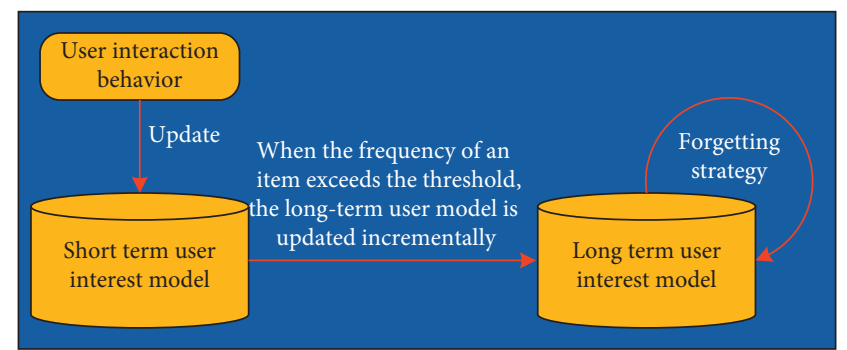

FIgURE 3: User interest model update.

network design method-with the goal of minimizing user travel time is required. The heuristic method includes four steps: network generation, network analysis, network optimization, and site setting, and then the feedback mechanism is introduced to gradually optimize the network design. After defining the user interest model, combined with the existing scenic spot database, the targeted tourism recommendation is formulated to form an effective tourism itinerary recommendation result. Travel itinerary recommendation consists of scenic spot recommendation and itinerary planning.

As shown in Figure 4, scenic spot recommendation is the basis of tourism itinerary planning, which determines the formulation of other decisions of tourism itinerary planning. Tourism is not a frequent event and has high real time, so the experiment designed a user travel destination recommendation algorithm based on multidimensional scoring.

As shown in Figure 5, the user travel destination recommendation algorithm based on multidimensional scoring includes scenic spot attribute scoring and user demand model scoring. The scenic spot attribute score consists of static attribute score and dynamic attribute score, in which the static score covers the scenic spot historical tourist score and geographical location; the dynamic score is based on the dynamic related information of scenic spot recommendation, including season and weather. In the tourist history evaluation of scenic spots, the score of 1-5 reflects the tourist 


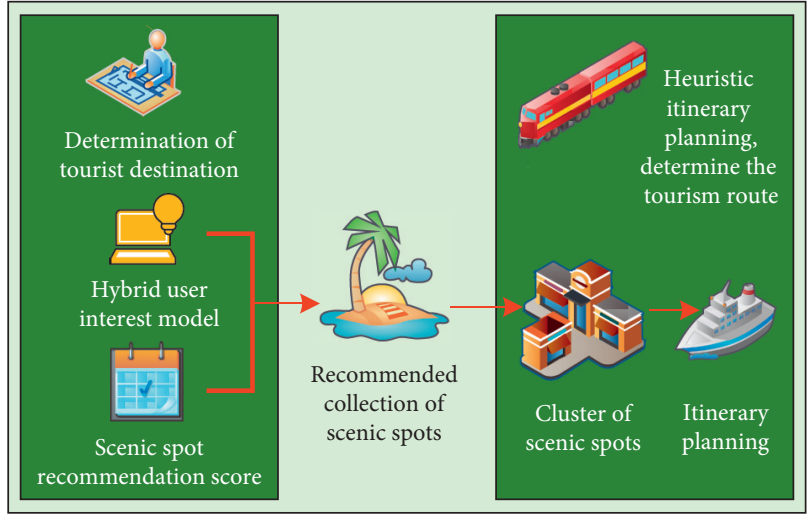

FIGURE 4: Overall structure of recommendation algorithm.

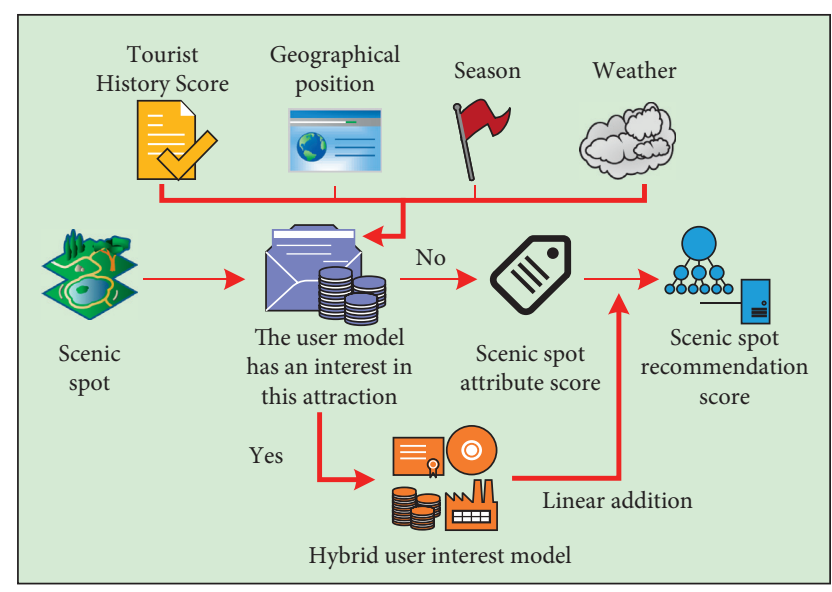

Figure 5: Composition of scenic spot recommendation score.

satisfaction. The higher the score, the higher the tourist satisfaction. Suppose there is a scenic spot history evaluation $\langle r 1, r 2, r 3, r 4, r 5\rangle$, with the number of users in the scenic spot history evaluation $i$ being $r i, i \in[1,2,3,4,5]$.

$$
H_{s}=\frac{\sum_{i=1}^{i=5} i * r_{i}}{5 * \sum_{i=1}^{i=5} r_{i}} .
$$

In equation (9), $H_{s}$ is the score of scenic spots in the historical evaluation, and its value is $0-1$. The distance between scenic spots is judged by the distance from the user's current location to the scenic spot. The closer the distance is, the lower the travel cost of the user is, and the more worthy of recommendation the scenic spot is. Let all the scenic spots around the current point $L$ be in the set $A(A 1, A 2, \ldots, A n)$, and calculate the distance $D_{i}$ from all the scenic spots $A i$ to point $\mathrm{L}$. The smaller the $D_{i}$, the higher the recommended value.

$$
D_{s}=\frac{\operatorname{Avg} D}{\operatorname{Avg} D+D_{i}}
$$

Equation (10) is the expression of the distance based rating $D_{s}$ of scenic spots. The average distance between scenic spots and $L$ points in the set $A(A 1, A 2, \ldots, A n)$ is $\operatorname{Avg} D$, which is $0-1$ and $\operatorname{Avg} D=\sum_{i=1}^{i=5} D_{i} / \sum_{i=1}^{i=5} i$. According to the matching degree of the season and the most suitable season, the scenic spot season is scored. When the matching degree is high, the scenic spot season score is $S e_{s}=1$; otherwise, $S e_{s}=0$. When the current weather condition is suitable for scenic spots, the weather score of scenic spots is $C_{s}=1$; otherwise, $C_{s}=0$.

$$
S=a * H_{s}+D_{s}+S e_{s}+C_{s} .
$$

Equation (11) is the attribute score of scenic spots obtained by integrating the four dimensions, $S$; $a$ value represents the weight of historical evaluation of scenic spots and is determined by the number of historical evaluations of scenic spots. The higher the number of evaluations of scenic spots, the higher the reliability of the score, and the greater the weight of historical evaluation in scenic spots.

$$
a_{i}=\frac{c_{i}}{\operatorname{Max}\left(c_{i}\right)} .
$$

In equation (12), the total number of historical evaluations of scenic spots is $c_{i}$, and the total number of historical evaluations of all scenic spots around the current point $L$ is $\operatorname{Max}\left(c_{i}\right)$.

$$
c_{i, j}=a S_{j}+b U_{i, j} .
$$

Formula (13) is the calculation formula of all the recommended scenic spots in the scenic spot attribute score set, $S$; the scenic spot attribute score of current scenic spot $j$ is $S_{j}$, and the interest value of user $i$ to scenic spot $j$ is $U_{i, j}$; when there is no interest value, $U_{i, j}=0 ; a$ and $b$ are adjustment coefficients. According to the recommendation score, the scenic spots in $S=\left(S_{1}, S_{2}, \ldots, S_{n}\right)$ are sorted. According to the multidimensional hybrid recommendation algorithm, the overall framework of the tourism recommendation system is constructed, which is mainly responsible for the recommendation of tourist attractions and tourism itinerary planning.

As shown in Figure 6, the experimental design of tourism recommendation system is composed of cloud server, user client, and related database. Among them, the cloud server is responsible for the interactive travel recommendation of the system client users, according to the user information and the corresponding scenic spot information in the database, and achieves the interactive task between the database and the corresponding user client; the database is responsible for storing the user information and the related scenic spot information; the user client can interact with the user, accept the user input, and push the most appropriate schedule through the visualization system.

\section{The Application Effect of Multidimensional Hybrid Recommended Travel Itinerary Planning Algorithm}

\subsection{Performance Verification of Multidimensional Hybrid} Travel Itinerary Recommendation Algorithm. Multidimensional hybrid travel recommendation starts from the user's interest range, with the purpose of meeting the user's needs, gaining satisfaction, and making scientific and effective travel plan. The calculation method of users' 


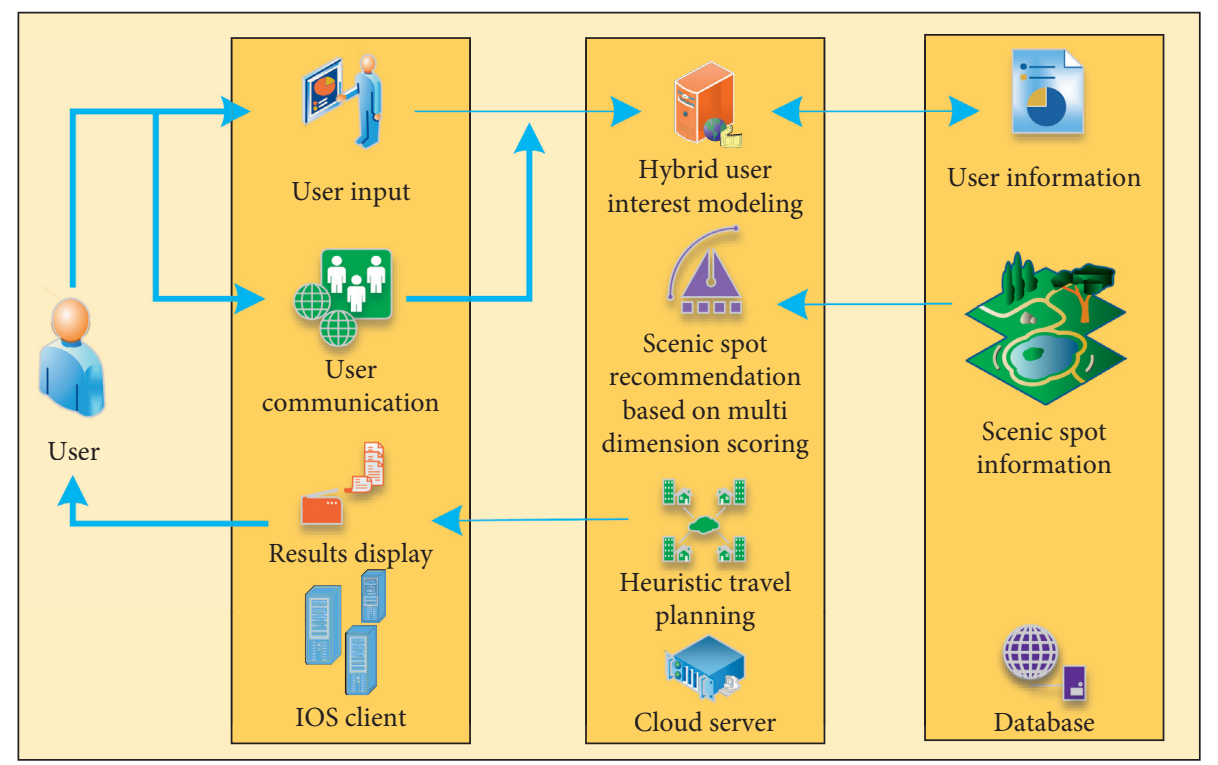

Figure 6: Overall structure of the system.

interest measurement of multidimensional features solves the problems of data sparsity and recommendation efficiency. This calculation method can adapt to both extremely sparse data sets and large data sets and improve the adaptability and scalability of the recommended model. This paper selects user based collaborative filtering recommendation algorithm (UB-CF), geographic location modeling recommendation algorithm (GEO-INFO), friend relationship based recommendation algorithm (SNS-INFO), and multidimensional hybrid travel itinerary recommendation algorithm $(\mathrm{MH})$ as the experimental objects to compare the accuracy and missing rate of the four travel itinerary planning algorithms.

As can be seen from Figure 7(a), the accuracy of the proposed multidimensional hybrid travel itinerary recommendation algorithm $(\mathrm{MH})$ is 0.984 , the accuracy of user based collaborative filtering recommendation algorithm (UB-CF) is 0.823 , the accuracy of GEO-INFO is 0.606 , and the accuracy of SNS-INFO is 0.278 . Figure $7(\mathrm{~b})$ shows that the missing rate of $\mathrm{UB}-\mathrm{CF}$ is 0.26 ; the missing rate of SNSINFO is 0.73 , being the highest; and the missing rate of multidimensional hybrid travel itinerary recommendation algorithm is 0 . To sum up, compared with the accuracy of SNS-INFO recommendation algorithm, the accuracy of multidimensional hybrid travel itinerary recommendation algorithm is improved by 0.706 , and the missing rate is reduced by 0.73 , which indicates that the travel itinerary planning completed by multidimensional hybrid travel itinerary recommendation algorithm can accurately meet the needs of users for travel itinerary planning and formulate personalized travel strategies in line with users, which is conducive to improving the quality of travel customer satisfaction.

Users are affected by both scenic spot information and geospatial information on their way to travel. Therefore, travel itinerary planning needs to analyze large-scale heat data and large-scale geospatial data to exclude the recommended single scenic spot with extremely short stay time. In addition, when the distance between scenic spots is too small, tourists often regard it as a scenic spot, so we should analyze the effect of multidimensional hybrid recommendation algorithm in scenic spot extraction. In the experiment, 215 scenic spots were given, and the demand time was increased by 50 minutes. The heat value Fr, utilization time ratio (UTR), stay time ratio (STR), and running time of four recommendation algorithms (Trip-Mine algorithm, mostbenefit algorithm, venuelnserting algorithm, and multidimensional hybrid travel recommendation algorithm) were compared.

Figure 8(a) shows that with the increase of constraints, the execution time of the algorithm is also gradually increasing, the number of tourist attractions on each path is increasing, and the number of user selectable paths is increasing. Compared with Trip-Mine algorithm, multidimensional hybrid travel itinerary recommendation algorithm has less execution time. When the time limit is $450 \mathrm{~min}$ and $500 \mathrm{~min}$, the running time of multidimensional hybrid travel recommendation algorithm is significantly reduced. Figure 8 (b) shows that the performance of multidimensional hybrid travel itinerary recommendation algorithm is better than that of mostbefifit algorithm and venuelnserting algorithm in travel itinerary planning. Figures $8(\mathrm{c})$ and $8(\mathrm{~d})$ show that in terms of utilization time ratio (UTR) and residence time ratio (STR) of tourism itinerary planning, the multidimensional hybrid tourism itinerary recommendation algorithm is significantly better than the other three algorithms; that is to say, the multidimensional hybrid tourism itinerary recommendation algorithm can make better use of the time so that users can arrange and make tourism itinerary planning schemes with higher timeliness ratio. To sum up, when the number of scenic spots is the same and the time limit is different, the multidimensional hybrid travel recommendation algorithm is better than the other three algorithms in terms of 


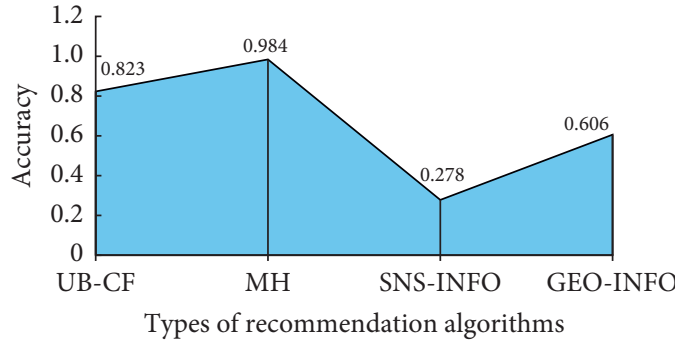

(a)

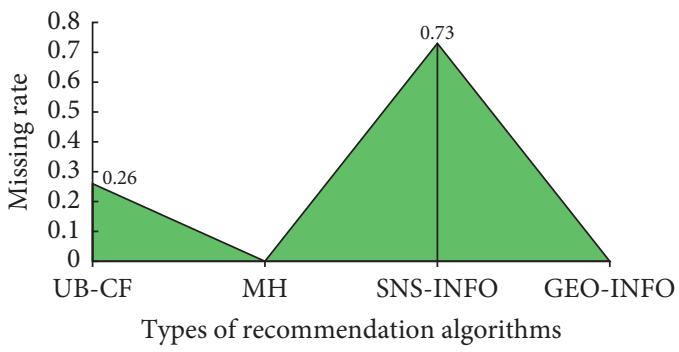

(b)

FIGURE 7: Comparison of accuracy and missing rate of four travel itinerary planning algorithms. (a) Accuracy of different recommendation algorithms. (b) Missing rate of different recommendation algorithms.

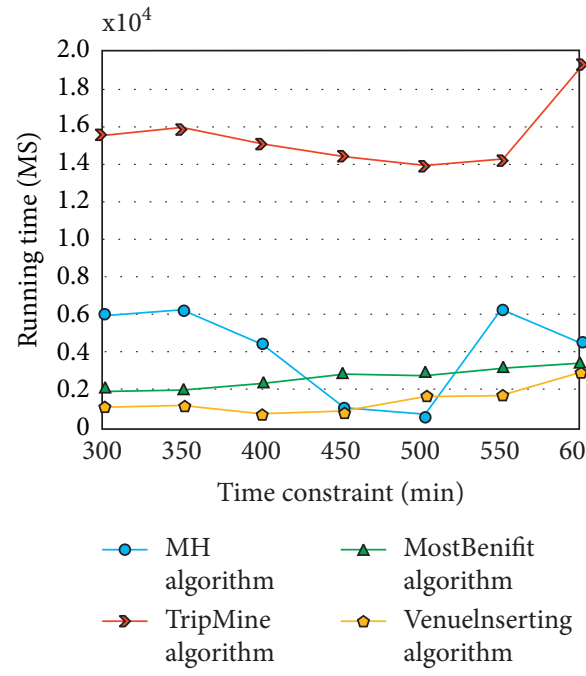

(a)

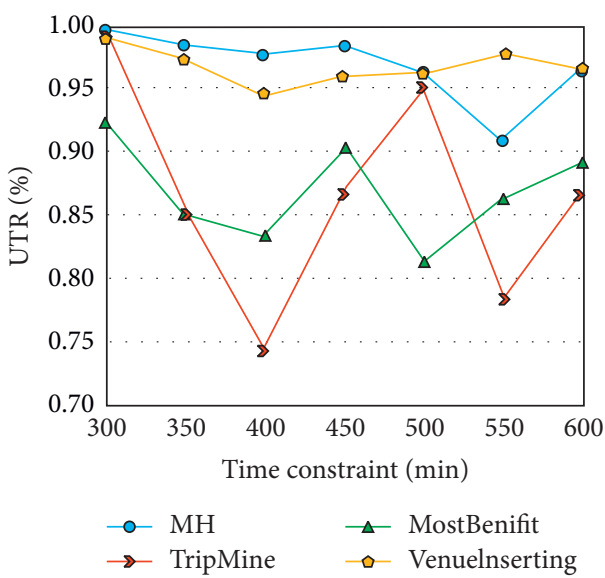

(c)

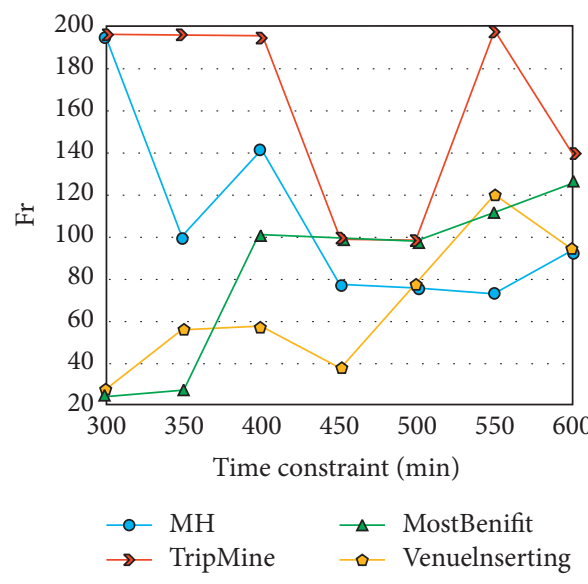

(b)

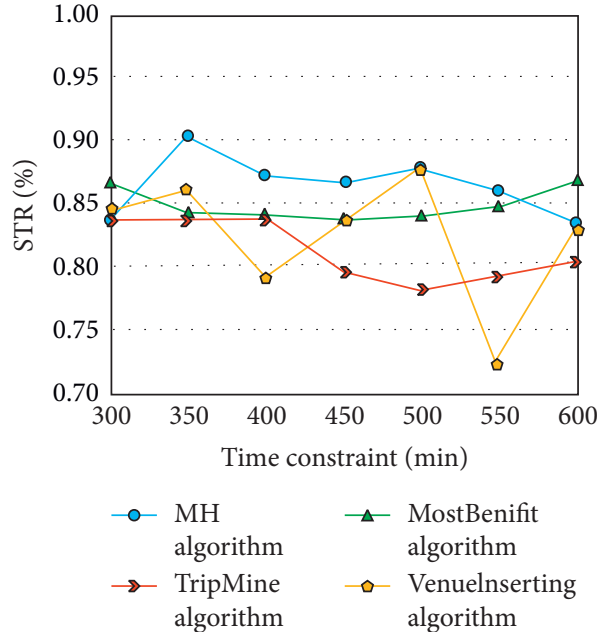

(d)

FIgURE 8: Experimental results under the same number of attractions and different time constraints. (a) Running time. (b) Fr value. (c) UTR value. (d) STR value.

UTR and STR value of travel planning. From the perspective of heat value Fr, the multidimensional hybrid travel recommendation algorithm is better than mostbenifit algorithm and venuelnserting algorithm in travel planning. Therefore, the multidimensional hybrid travel recommendation algorithm is better than the other three algorithms in running time when the time limit meets the expectation. 


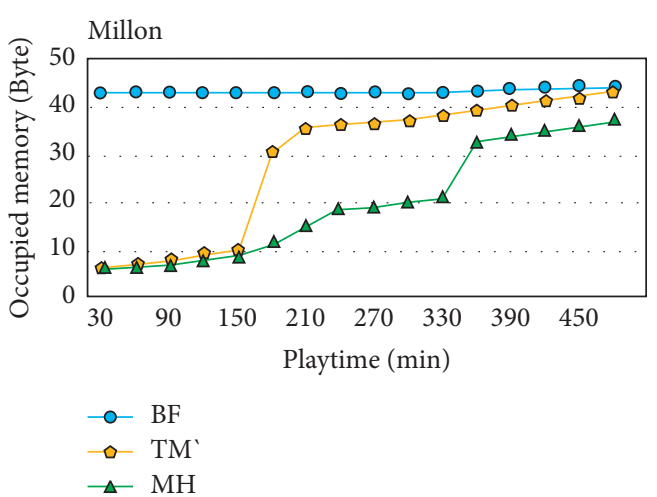

(a)

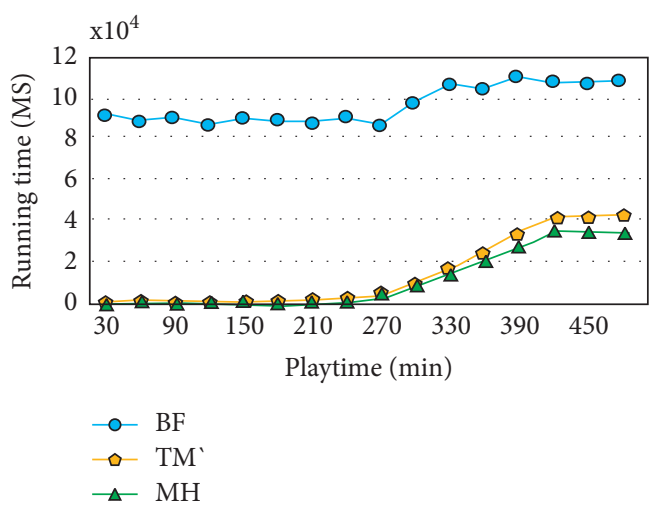

(c)

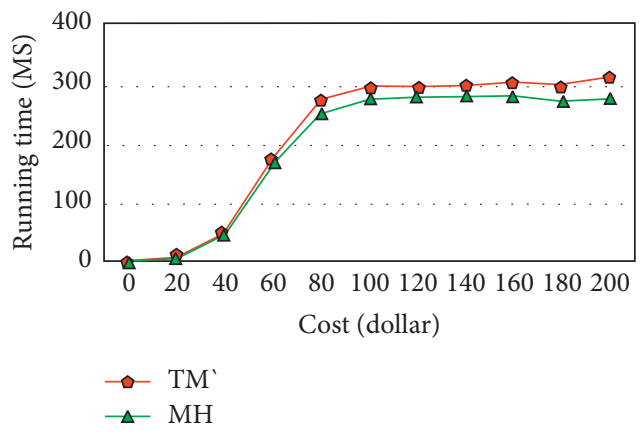

(b)

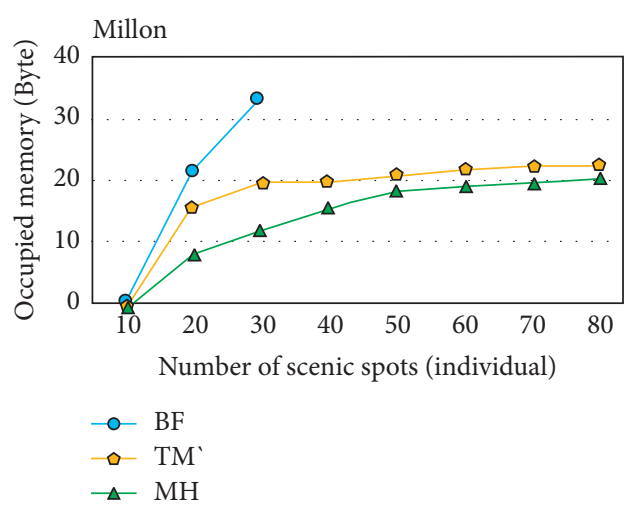

(d)

Figure 9: Time ratio comparison line chart. (a) Memory proportion of different play time. (b) Comparison of running time under different costs. (c) Running time under playing time. (d) Comparison of the number of different scenic spots.

Figure 9(a) shows the comparison results of memory consumption of different algorithms with different time, the same cost, and the same number of scenic spots. When the travel time is less than $150 \mathrm{~min}$, the memory proportion of TM (Trip-Mine) algorithm and multidimensional hybrid travel itinerary recommendation algorithm $(\mathrm{MH})$ is similar. When the travel time is $180 \mathrm{~min}$, the memory occupation of TM algorithm increases rapidly until it is the same as BF (brute force) algorithm, which is stable at about $45 \mathrm{~m}$; the memory occupation of $\mathrm{MH}$ algorithm increases, and the slope comes at $360 \mathrm{~min}$. Figure 9(b) shows the running time comparison of TM algorithm and $\mathrm{MH}$ algorithm when the cost is different and the number of scenic spots and travel time are the same. The running time of the two algorithms is very close before the tourism cost is 40 US dollars. With the increase of the cost, the running time of $\mathrm{MH}$ algorithm is always less than that of TM algorithm. Figure 9(c) shows the running time comparison results of $\mathrm{BF}$ algorithm, TM algorithm, and $\mathrm{MH}$ algorithm with different travel time, the same travel cost, and the same number of scenic spots. The running time of $\mathrm{BF}$ algorithm is basically not affected by the travel time, and it is stable at about 1 million milliseconds. With the increase of travel time, the running time of TM algorithm and $\mathrm{MH}$ algorithm increases. When the time exceeds 330 hours, the running time of $\mathrm{MH}$ algorithm is gradually less than TM algorithm, and the running time of $\mathrm{MH}$ algorithm is less than $1 / 5$ of that of TM algorithm. This means that with the increase of travel time, $\mathrm{MH}$ algorithm will show better performance. Figure 9(d) shows the comparison results of memory occupation of $\mathrm{BF}$ algorithm, TM algorithm, and $\mathrm{MH}$ algorithm when the number of scenic spots is different and the travel cost and travel time are the same. When the number of scenic spots is 20 to 30 , the memory consumption of $\mathrm{MH}$ algorithm is only $1 / 2$ of that of $\mathrm{TM}$ algorithm. With the increase of the number of scenic spots, the memory consumption of $\mathrm{MH}$ algorithm is always lower than that of TM algorithm. From the above results, we can see that the multidimensional hybrid travel itinerary recommendation algorithm is efficient.

4.2. Analysis of the Application Effect of Tourism Itinerary Planning System. Select 50 user's travel strategies about $\mathrm{H}$ city; form data set $s$, extract relevant scenic spots information; use multidimensional scenic spot scoring method to score related scenic spots; use heuristic itinerary planning algorithm to plan travel routes; calculate the travel time of scenic spots and the total distance between scenic spots; calculate the time efficiency ratio (the ratio of travel time to the total distance spent between scenic spots). The higher the time ratio is, the better the route planning is.

In Figure 10, " $P$ " refers to the original tourism strategy, and " $P$ *" refers to the tourism strategy formulated by heuristic itinerary planning, so "distance $(P)$ " refers to the 


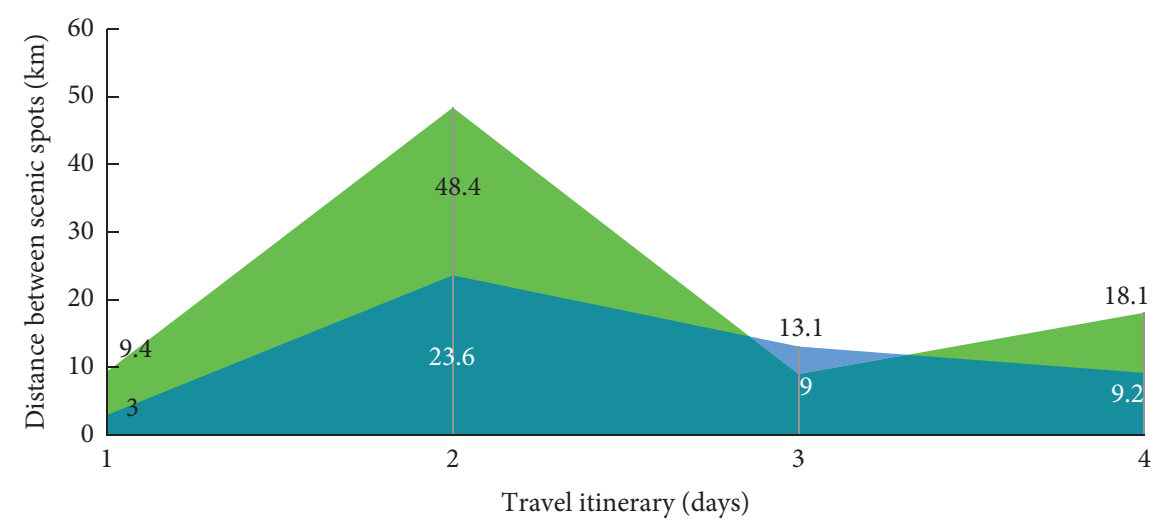

Distance $(\mathrm{P})$

Distance $\left(\mathrm{P}^{*}\right)$

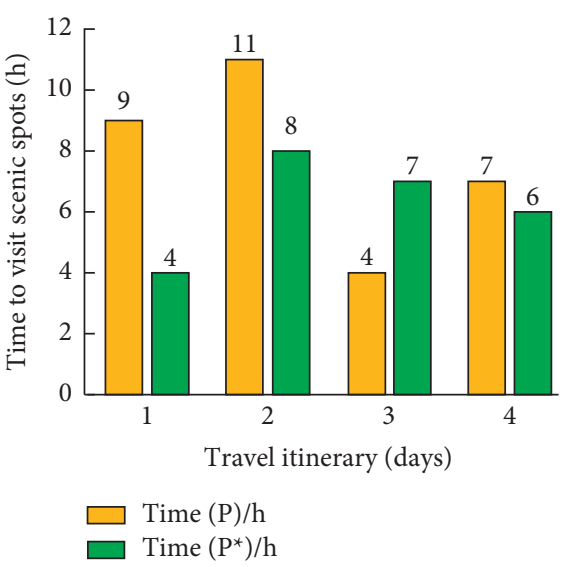

(b)

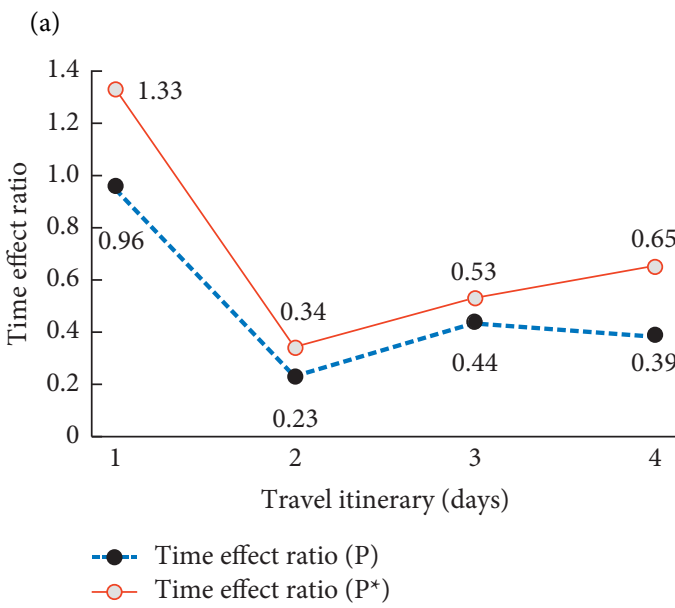

(c)

FIGURE 10: Comparison between original tourism strategy and heuristic itinerary planning strategy. (a) Change of distance between tourist attractions. (b) Time changes of scenic spots. (c) Change of aging ratio.

distance between scenic spots in the original tourism strategy, and "distance $(P *)$ " refers to the distance between scenic spots in the heuristic itinerary planning tourism strategy. "Time" refers to the time of scenic spots. As can be seen from Figure 10, in the travel itinerary planned by the multidimensional hybrid recommendation algorithm, the total distance between scenic spots is $48.9 \mathrm{~km}$, and the total time of visiting scenic spots is $25 \mathrm{~h}$; in the original travel strategy, the total distance between scenic spots is 84.9 , and the total time of visiting scenic spots is $31 \mathrm{~h}$. The multidimensional hybrid recommendation algorithm can significantly improve the time efficiency ratio of tourists' travel itinerary planning and increase it from 0.37 (in the original travel strategy) to 0.51 . The travel planning itinerary obtained by the hybrid recommendation algorithm is significantly better than the route planning of the original tourism strategy, and the daily arrangement of scenic spots is more reasonable, which can help tourists reduce the travel and time between scenic spots.

Figure 11 shows that, from the overall analysis, the time efficiency ratio of the travel route planned by the heuristic travel planning algorithm (multidimensional hybrid recommendation algorithm) is significantly better than that of the original travel strategy, which shows that the multidimensional hybrid recommendation algorithm designed in the experiment has certain effectiveness in the travel planning of tourists. When the scenic spots and travel days remain unchanged, the multidimensional hybrid recommendation algorithm is effective travel itinerary recommendation algorithm and can effectively reduce the distance tourists spend between scenic spots, significantly improve the travel time ratio, and get more uniform and reasonable travel planning.

After setting the destination and the starting place, the departure time, and the return time, the tourists design the travel planning system with the multidimensional hybrid travel recommendation algorithm as the core component. According to the user's choice, the personalized travel planning scheme will be made for the user. Figure 12 shows only part of the itinerary planning route. According to the tourism itinerary planning system, users can select and click their own regions of interest for online browsing of scenic spots and real-time adjustment of the planning route. 


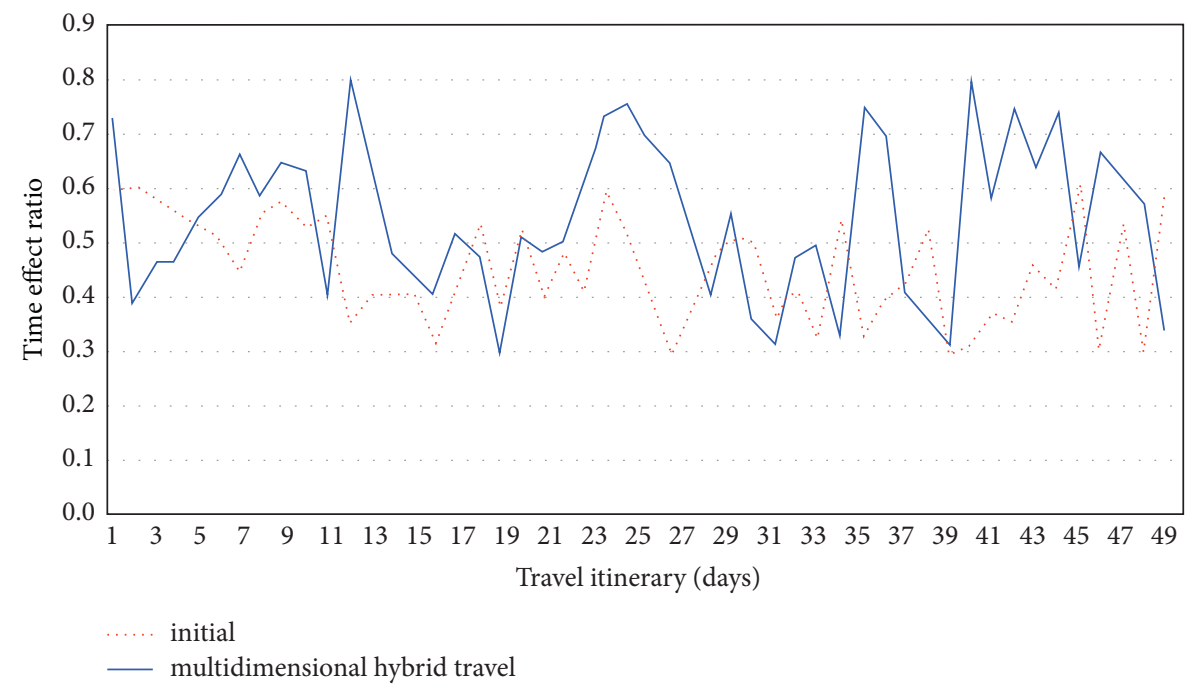

Figure 11: Time ratio comparison line chart.

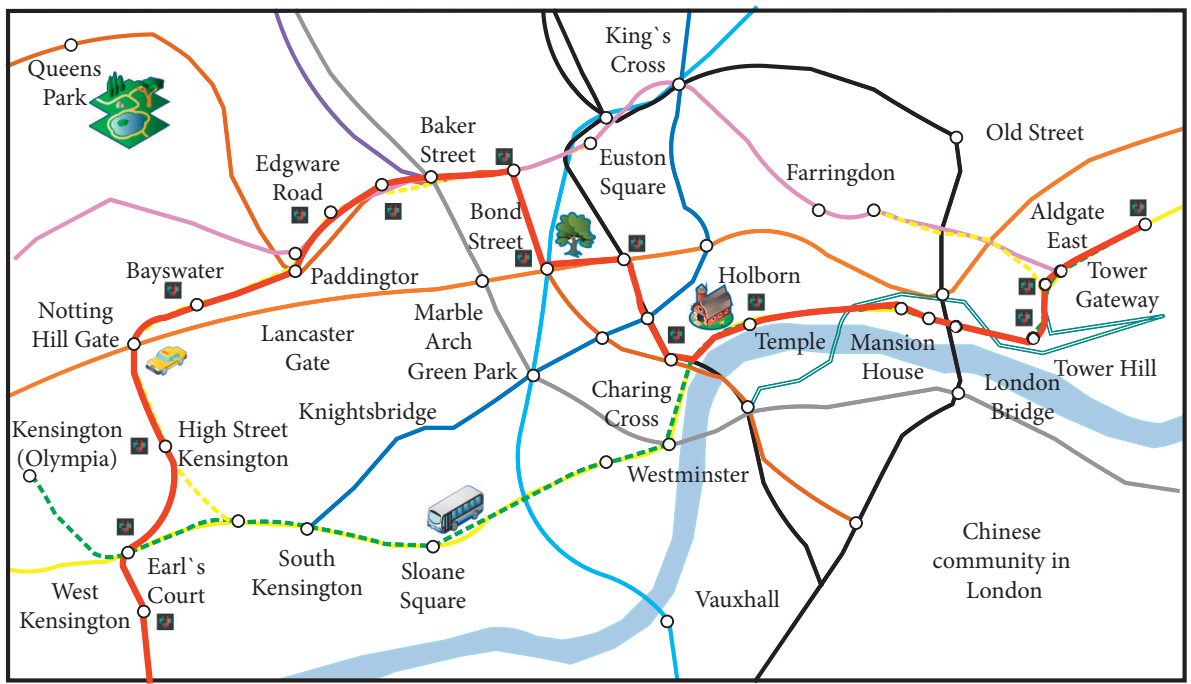

FIGURE 12: Tourism itinerary planning scheme.

\section{Conclusion}

With the improvement of living standards and the rapid development of the tourism industry, tourists often obtain tourism related information through Internet search. However, the amount of tourism information on the Internet often causes the problem of user information overload, which leads to the users being unable to accurately obtain the tourism information they are interested in. In order to plan a scientific and efficient travel itinerary, a travel itinerary planning system based on multidimensional hybrid travel itinerary recommendation algorithm is designed. In terms of accuracy, the accuracy of multidimensional hybrid travel itinerary recommendation algorithm is 0.984 and the missing rate is 0 ; the accuracy of UB-CF algorithm, GEO-INFO algorithm, and SNS-INFO algorithm is 0.823, 0.606, and 0.278 , respectively, and the missing rate is $0.26,0$, and 0.73, respectively. Compared with Trip-Mine algorithm, the running time of multidimensional hybrid travel itinerary recommendation algorithm is significantly shorter. When the time limit is $450 \mathrm{~min}$ and $500 \mathrm{~min}$ in UTR and STR, the multidimensional hybrid travel recommendation algorithm is superior to mostbenifit algorithm and venuelnserting algorithm. When the travel cost and travel time are the same and the number of scenic spots is 20 to 30 , the memory occupation of $\mathrm{MH}$ algorithm is only half that of TM algorithm. The multidimensional hybrid recommendation algorithm can significantly improve the time efficiency of tourist travel planning, increasing it from 0.37 (in the original travel strategy) to 0.51. To sum up, the multidimensional hybrid travel recommendation algorithm can accurately meet the needs of users for travel planning and formulate personalized travel strategies. However, the research of this paper also has some limitations. When establishing the mixed user interest model, the information collection of users of different tourism route planning systems is insufficient, 
resulting in the incomplete interest model. Therefore, in the future, we will also conduct in-depth exploration of user information collection from this aspect.

\section{Data Availability}

The experimental data used to support the findings of this study are available from the corresponding author upon request.

\section{Conflicts of Interest}

The authors declare that they have no conflicts of interest regarding this work.

\section{Acknowledgments}

This work was supported by 2021 Research Project on the Development of Social Sciences in Hebei Province, "Research on the Action Mechanism of Tourism Network Public Opinion Influencing Factors in the Post-epidemic Era," Project No. 202102012162020; 2021 Research Project on the Development of Social Sciences in Hebei Province, "Research on Supply and Demand Matching and Guarantee Mechanism of Ice and Snow Industry Development in Hebei Province," Subject No. 20210201229; and 2020 Qinhuangdao Science and Technology Research and Development Plan, "Research on Regional Cooperation Mechanism of Ice and Snow Tourism in Qinhuangdao from the Perspective of Space Network," Subject No. 202004A146.

\section{References}

[1] C. Koo and L. Cantoni, "Special issue on informatics/data analytics in smart tourism," Information Processing \& Management, vol. 55, no. 4, pp. 1373-1375, 2019.

[2] A. E. Arenas, J. M. Goh, and A. Uruena, "How does IT affect design centricity approaches: evidence from Spain's smart tourism ecosystem," International Journal of Information Management, vol. 45, no. APR, pp. 149-162, 2019.

[3] J. Y. Kim, N. Chung, and K. M. Ahn, "The impact of mobile tour information services on destination travel intention," Information Development, vol. 35, no. 1, pp. 107-120, 2019.

[4] C. D. Huang, J. Goo, K. Nam, and C. Woo Yoo, "Smart tourism technologies in travel planning: the role of exploration and exploitation," Information \& Management, vol. 54, no. 6, pp. 757-770, 2017.

[5] Z. Liu, J. Cao, Y. Tan, Q.-W. Xiao, and M. Prasad, "Planning above the API clouds before flying above the clouds: a realtime personalized air travel planning approach," International Journal of Parallel Programming, vol. 48, no. 1, pp. 137-156, 2020.

[6] P. Yochum, L. Chang, T. Gu, and M. Zhu, "An adaptive genetic algorithm for personalized itinerary planning," IEEE Access, vol. 8, 2020.

[7] G. Zhu, Y. Wang, J. Cao et al., "Neural attentive travel package recommendation via exploiting long-term and short-term behaviors," Knowledge-Based Systems, vol. 211, Article ID 106511, 2021.

[8] J. Jiao, X. Zhang, F. Li, and F. Wang, "A novel learning rate function and its application on the SVD++ recommendation algorithm," IEEE Access, vol. 8, pp. 14112-14122, 2020.
[9] L. Ravi, V. Subramaniyaswamy, V. Vijayakumar, S. Chen, A. Karmel, and M. Devarajan, "Hybrid location-based recommender system for mobility and travel planning," Mobile Networks \& Applications, vol. 24, no. 4, pp. 1226-1239, 2019.

[10] A. S. M. Arif and J. T. Du, "Understanding collaborative tourism information searching to support online travel planning," Online Information Review, vol. 43, no. 3, pp. 369-386, 2019.

[11] B. Qu, W. Yang, G. Cui, and X. Wang, "Profitable taxi travel route recommendation based on big taxi trajectory data," IEEE Transactions on Intelligent Transportation Systems, vol. 21, 2019.

[12] D. Lyu, L. Chen, Z. Xu, and S. Yu, "Weighted multi-information constrained matrix factorization for personalized travel location recommendation based on geo-tagged photos," Applied Intelligence, vol. 50, no. 1, pp. 1-15, 2020.

[13] M. N. Sweet, "User interest in on-demand, shared, and driverless mobility: evidence from stated preference choice experiments in Southern Ontario," Travel Behaviour and Society, vol. 23, pp. 120-133, 2021.

[14] D. Liu, Z. Wang, and J. Zhang, "Video stream distribution scheme based on edge computing network and user interest content model," IEEE Access, vol. 8, pp. 30734-30744, 2020.

[15] H. Li, H. Duan, Y. Zheng, Q. Wang, and Y. Wang, "A CTR prediction model based on user interest via attention mechanism," Applied Intelligence, vol. 50, no. 4, pp. 1192-1203, 2020.

[16] Q. Wang, F. Liu, P. Huang, S. Xing, and X. Zhao, "A hierarchical attention model for CTR prediction based on user interest," IEEE Systems Journal, vol. 14, no. 3, pp. 4015-4024, 2020.

[17] M. Xu and S. Liu, "Semantic-enhanced and context-aware hybrid collaborative filtering for event recommendation in event-based social networks," IEEE Access, vol. 7, pp. 17493-17502, 2019.

[18] X. Feng, D. Zhong, and H. Yu, "A clustering algorithm based on emotional preference and migratory behavior," Soft Computing, vol. 24, no. 10, pp. 7163-7179, 2020.

[19] H. Aziz, P. Biro, S. Gaspers, R. de Haan, N. Mattei, and B. Rastegari, "Stable matching with uncertain linear preferences," Algorithmica, vol. 82, no. 5, pp. 1410-1433, 2020. 